\title{
The great ozone controversy
}

\author{
While new data on the Antarctic springtime ozone hole does not bear directly on the problem of \\ ozone destruction worldwide, it does significantly shift the balance of belief.
}

General impatience with the process of science will be moderately enhanced by the important article on ozone in the Antarctic atmosphere by C.B. Farmer and his colleagues on page 126 of this issue. Farmer's group has made the first accurate measurements of the presence of simple halogen compounds in the springtime ozone hole in the Antarctic stratosphere, yet go no further than to say that their observations are "entirely consistent" with the hypothesis that there is a cause and effect relationship between the presence of the halogens and the absence of ozone. Even J.C.Farman and J.A.Pyle, writing less formally, go no further than to say that they believe the connection is causal, but in language suggesting that dissenters are free to believe differently.

Why, when professionals cannot make up their minds even about what is happening in the Antarctic, should the world's diplomats be locked in negotiation of the fine print of the Vienna Convention on the Preservation of the Ozone Layer? That is what a great many outsiders (not to mention some interested parties) will be asking. Do chlorofluorohydrocarbons deplete the atmosphere of ozone, or do they not? And if not, why all the fuss?

Yet circumstances like these are common. It is now more than thirty years since the demonstration (using British data) by Bradford-Hill and Doll of a strong correlation between cigarettesmoking and the occurrence of lung cancer, but a large part of that interval has been occupied with arguments about the likelihood that the connection could be causal.

Indeed, the passage of time does suggest that the relationship may be more complicated than it seemed at first. There may be genetic factors. It could be that cigarettes are blackened in the eyes of potential users by environmental influences not recognized at the outset (the previously unsuspected high concentration of radon in some parts of the world, for example). Yet what survives through thirty years of argument is that the correlation is so strong, and its consequences statistically so steady, that doubts about the reality of the correlation are melting away.

So shall we have to wait for thirty years before knowing what these chemicals do to ozone in the stratosphere, and find then that we all have skin cancer? Should not problems of cause and effect be simpler in the physical sciences, where it is possible to measure things with arbitrary precision, where the variations between the entities observed are not as great as they are among people and where the causal links are derived from immutables such as Newton's laws?

The simple riposte is that there are many people working in the observational physical sciences who wish that were indeed the case. Ask any physical oceanographer or meteorologist.

Ozone measurements have been plagued with difficulty since they were first begun by Dobson, at Oxford, in the $1930 \mathrm{~s}$. For much of the interval, there have been instrumental difficulties, only satisfactorily resolved in the past ten years with the development of sensitive solidstate infrared detectors for recording the flux at the ground (or elsewhere) of solar radiation at the absorption frequency of ozone.

Then there are problems of interpretation. How to distinguish between the ozone layer in the lower stratosphere and ozone in the troposphere? Are fluctuations in time, on a timescale of half an hour or so, a sign that stratospheric ozone is patchy or of some temporary imbalance in the competing processes of formation and destruction at high altitudes? Does the elevation of the line of sight to the Sun matter?

At various times, all these effects have been offered as explanations of puzzling measurements. Ironically, whatever may be the effect of manmade chemicals on the ozone in the lower stratosphere, it is probable that activities nearer the surface of the Earth, by increasing the amount of low-level ozone, have complicated the problem of using Dobson intruments to tell the secular trend of ozone in the atmosphere. In principle, satellite instruments using the ultraviolet reflectance of the atmosphere should avoid many of these difficulties by giving a more synoptic measurement, but their sensitivity is not yet as great.

Farmer's measurements are in some ways more easily interpreted. The springtime decline of ozone in Antarctica was first recognized from historical records from Halley Bay (J.C.Farman, B. G. Gardiner \& J.D.Shanklin, Nature 315, $207 ; 1985)$ and on the basis of measurements in the spring of 1984 (September and October). Since then, there have been only two Antarctic springs (the third is about to begin).

Farmer and his colleagues (also based at Halley Bay) were lucky last year to find themselves alternatively outside and inside the polar vortex that seems to keep a column of polar atmosphere as an almost isolated air mass for months on end. What they find is that there are high concentrations of simple halogen molecules in the Antarctic atmosphere, which may not surprise those who have always believed that halogenated hydrocarbons are the death of ozone, but that the properties of the column within the polar vortex differ surprisingly from expectation.

With hindsight, it is easy to believe that the cold atmosphere above the winter pole would be a a physical sink for halogenated hydrocarbons such as those used in aerosol cans and refrigerators. Pyle and Farman raise the interesting question of the form in which these materials are condensed before being made gaseous, and destructive of ozone, by the Sun's return. Others will raise the possibility that the winter poles may be more permanent sinks for them, places from which they are removed from the low winter stratosphere by precipitation, and will go on collecting snow for analysis from the Antarctic.

Meanwhile, the grounds for believing that halogenated hydrocarbons destroy ozone are changed in only one respect, important though that may be. The striking correlation between low concentration of ozone and high concentrations of halogen compounds in one exceptional part of the atmosphere, coupled with the expectation from laboratory results that the former should be destroyed by the latter, is the equivalent in this connection of a demonstration in biology that a process observed in vitro occurs in vivo as well. So it is natural to believe that the ozone hole has been explained. That is what Farman and Pyle say.

But nobody, for the time being, has direct evidence that the same materials destroy ozone globally. If the winter pole takes halogenated hydrocarbons permanently out of circulation, it might thus ameliorate what happens elsewhere. But that this behaviour might compensate for the annual discharge of chemicals to the atmosphere is against the odds. The diplomats had better keep talking.

John Maddox 